Hum Genet (1993) 92:527-532

\title{
A strategy for the characterization of minute chromosome rearrangements using multiple color fluorescence in situ hybridization with chromosome-specific DNA libraries and YAC clones
}

\author{
Susanne Popp ${ }^{1}$, Anna Jauch ${ }^{1}$, Detlev Schindler ${ }^{2}$, Michael R. Speicher ${ }^{1}$, Christoph Lengauer ${ }^{1}$, \\ Helen Donis-Keller ${ }^{3}$, Harold C. Riethman ${ }^{4}$, Thomas Cremer ${ }^{1}$ \\ ${ }^{1}$ Institut für Humangenetik und Anthropologie der Universität Heidelberg, Im Neuenheimer Feld 328, D-69120 Heidelberg, Germany \\ ${ }^{2}$ Institut für Humangenetik, Biozentrum der Universität Würzburg, Am Hubland, D-97074 Würzburg, Germany \\ ${ }^{3}$ Washington University School of Medicine, Division of Human Molecular Genetics, Department of Surgery, \\ 660 S. Euclid, St. Louis, MO 63110 , USA \\ ${ }^{4}$ The Wistar Institute, 3601 Spruce Street, Philadelphia, PA 19104, USA
}

Received: 4 March 1993 / Revised: 7 May 1993

\begin{abstract}
The identification of marker chromosomes in clinical and tumor cytogenetics by chromosome banding analysis can create problems. In this study, we present a strategy to define minute chromosomal rearrangements by multicolor fluorescence in situ hybridization (FISH) with "whole chromosome painting" probes derived from chromosome-specific DNA libraries and Alu-polymerase chain reaction (PCR) products of various region-specific yeast artificial chromosome (YAC) clones. To demonstrate the usefulness of this strategy for the characterization of chromosome rearrangements unidentifiable by banding techniques, an $8 p+$ marker chromosome with two extra bands present in the karyotype of a child with multiple anomalies, malformations, and severe mental retardation was investigated. A series of seven-color FISH experiments with sets of fluorochrome-labeled DNA library probes from flow-sorted chromosomes demonstrated that the additional segment on $8 \mathrm{p}+$ was derived from chromosome 6. For a more detailed characterization of the marker chromosome, three-color FISH experiments with library probes specific to chromosomes 6 and 8 were performed in combination with newly established telomeric and subtelomeric YAC clones from $6 \mathrm{q} 25$, $6 \mathrm{p} 23$, and $8 \mathrm{p} 23$. These experiments demonstrated a trisomy $6 \mathrm{pter} \rightarrow 6 \mathrm{p} 22$ and a monosomy 8 pter $\rightarrow 8 \mathrm{p} 23$ in the patient. The present limitations for a broad application of this strategy and its possible improvements are discussed.
\end{abstract}

Dedicated to Professor Dr. U. Wolf on the occasion of his 60 th birthday

Correspondence to: T. Cremer

\section{Introduction}

Fluorescence in situ hybridization (FISH) has become a powerful tool for studying chromosome aberrations in clinical and tumor cytogenetics (for a review, see Lichter et al. 1991). Until now, applications of FISH were most useful for the detailed evaluation of cases where chromosomes or chromosomal subregions of interest were previously known to the investigator or where chromosome banding analyses had provided a testable assumption of the genetic composition of a marker chromosome. In these cases, appropriate DNA probes could easily be chosen for FISH. Here, we describe a strategy to define the origin of genetic material in marker chromosomes even in cases where this origin cannot be resolved by using conventional chromosome analyses. This strategy is based on recent advances in multicolor chromosome painting (Nederlof et al. 1990, 1992; Ried et al. 1992a, b; Dauwerse et al. 1992; Lengauer et al. 1993).

The feasibility of this strategy is demonstrated for the karyotype of a child with a marker chromosome $8 \mathrm{p}+$, containing a chromosome segment of previously unknown origin. To avoid the time-consuming procedure of testing individual whole chromosome painting (WCP) probes, multiple color chromosome in situ suppression (CISS) hybridization was performed with sets of up to seven WCP probes simultaneously. In order to paint all 24 human chromosome types and to define the chromosomal origin of the segment in question, a maximum of four FISH experiments with appropriate combinations of WCP probes is required. FISH with yeast antificial chromosome (YAC) clones mapped to the chromosome in 
question can then be applied to define the chromosomal subregion.

\section{Materials and methods}

\section{Cell material}

Heparinized blood samples were obtained from a 9-year-old girl and from both parents. The girl shows a microcephalus and multiple other anomalies, including blepharoptosis, a persistent ductus Botalli, and stenosis of the pulmonal artery. Psychomotor development has been delayed and the girl is severely mentally retarded. A detailed description and discussion of the clinical symptoms will be presented elsewhere (D. Schindler et al., in preparation) taking into account the cytogenetic findings described below.

Metaphase spreads were prepared from phytohemagglutinin (PHA)-stimulated lymphocytes of the proband and both parents. Replication banding was carried out according to Dutrillaux et al. (1976).

\section{DNA probes}

Chromosome-specific plasmid libraries from sorted human chromosomes $1,4,8$, and 13 were kindly provided by Dr. J. Gray (University of California, San Francisco, CA) (Collins et al. 1991). The amplification of these libraries and isolation of plasmid DNA were carried out according to standard protocols (Sambrook et al. 1989). Biotin-labeled libraries specific for human chromosomes 6 and 8 . respectively, and generated by the polymerase chain reaction (PCR) of sorted chromosomes (Telenius et al. 1992), were kindly provided by AGS (Angewandte Gentechnologie Systeme, Heidelberg, Germany). Chromosome-specific library DNA probes directly labeled with fluorochromes, termed WCP probes, were a generous gift from Imagenetics (Naperville, IL). WCP probes for chromosomes $1,4,6$, and 9 conjugated to a fluorescein isothiocyanate (FITC) derivative (Spectrum Green) and for chromosomes $4,6,8,9$, and 12 conjugated to a rhodamine derivative (Spectrum Orange) were used. Three YAC clones mapped in our laboratory were used to define chromosome subregions of interest. Clone HTY3103 (human insert size approximately $450 \mathrm{~kb}$ ) maps to $6 \mathrm{p} 22 \rightarrow \mathrm{p} 23$ and 18q23; clone HTY3188 (approximately $250 \mathrm{~kb}$ ) maps to $6 \mathrm{q} 27$ and $8 \mathrm{p} 23$. Additional weaker signals were observed at $1 \mathrm{p} 36,1 \mathrm{q} 41 \rightarrow \mathrm{q} 42,1 \mathrm{q} 44,2 \mathrm{q} 37,5 \mathrm{q} 35$, and $11 \mathrm{p} 15$ (A. Jauch, T. Cremer, H. Donis-Keller, H. C. Riethman, unpublished data). Clone EG8 (human insert size not known) maps to bands $1 \mathrm{p} 32$, 1q32, and 6q25 (Lengauer et al. 1993).

\section{Probe labeling}

Alu-PCR products from YAC clones EG8, HTY3103, and HTY3188 were produced as described by Lengauer et al. (1992) and nick-translated with biotin-11-dUTP. Plasmid library DNAs from sorted human chromosomes $1,4,8$, and 13 were nick-translated with coumarin-4-dUTP (Fluoro-Blue, a generous gift from Amersham, Buckinghamshire, UK) (Lengauer et al. 1993).

\section{CISS hybridization and detection}

Prior to CISS hybridization, slides were pretreated as described by Ried et al. (1992c). CISS hybridization was carried out as described by Lichter and Cremer (1992) with the following modifications. For each probe, optimum amounts per slide (hybridized area $15 \times 15 \mathrm{~mm}$ ) were determined empirically. Imagenetics WCP probes were diluted $1: 10$ in $10 \mathrm{~m} M$ TRIS $\mathrm{HCl} / 1 \mathrm{~m} M$ EDTA, and $2.5-15 \mu \mathrm{l}$ of the diluted probe were used. Aliquots of $1-2 \mu \mathrm{g}$ of plasmid DNA libraries were applied. Biotin-labeled Alu-PCR products from YAC clone DNA were used at an amount of 80-150 ng. For combinatorial FISH, probes for various chromosomes were combined with or without YAC probes and precipitated in the presence of 100-150 $\mu \mathrm{g}$ Cot-1 DNA (Life Technologies, Eggenstein, Germany), 50-100 $\mu \mathrm{g}$ sonicated salmon sperm DNA (Sigma, Munich, Germany), and $300 \mathrm{mM}$ sodium acetate. Multiplex probes were resuspended in the hybridization mixture contained in the Imagenetics WCP probe kit. After 35-65 h incubation at $37^{\circ} \mathrm{C}$, slides were washed three times at $37^{\circ} \mathrm{C}$ in $2 \times \operatorname{SSC}(1 \times$ $\mathrm{SSC}=150 \mathrm{~m} M \mathrm{NaCl} / 15 \mathrm{~m} M$ sodium citrate, $\mathrm{pH} 7.0$ ), followed by a short wash under tap water; they were then air-dried and mounted in $0.1 \%$ p-phenylenediamindihydrochloride in glycerol (Johnson and Nogueira Araujo 1981). In experiments that included biotinylated YAC probes, slides were washed $3 \times 5 \mathrm{~min}$ in $50 \%$ formamide, $1 \times \mathrm{SSC}$ at $45^{\circ} \mathrm{C}$, and $5 \mathrm{~min}$ in $2 \times \mathrm{SSC}$ at $37^{\circ} \mathrm{C}$. Biotinylated probes were detected using avidin conjugated to fluorescein (Vector Laboratories, Heidelberg, Germany). In some experiments, signals from the biotinylated probes were amplified once as described by Pinkel et al. (1986).

\section{Conventional and digital fluorescence microscopy}

Cells were viewed with a Zeiss photomicroscope Axiophot equipped for epifluorescence, and with a Plan-Neofluar $63 x / 1.25$ oil objective. Microphotographs were taken on Ektachrome 400 ASA and Agfachrome 1000 ASA color-slide films. For triple exposures of color-slide films, the following filters (Carl Zeiss, Oberkochen, Germany) were used: filter set 10 (BP 450-490, FT 510, LP 515-565) for Spectrum Green or FITC, filter set 01 (BP 365 , FT 395, LP 397) for the coumarin derivative (Fluoro-Blue), and the double band pass filter set 23 for simultaneous Spectrum Green/FITC and Spectrum Orange detection (DBP 485/20, 546/12; FT 500/560; DBP 515-530, 580-630).

For digital fluorescence microscopy, the Axiophot was coupled to a cooled charge-coupled device (CCD) camera (Photometrics, Munich, Germany, equipped with a Kodak KAF 1400 chip). A Macintosh Quadra 900 was used for camera control and digital image acquisation in the "TIFF" format using the software package Nu200 2.0 (Photometrics). Separate gray scale fluorescence images were recorded for each fluorochrome. In addition to filter set 09 , a filter set consisting of BP 365, FT 395, and BP 450-490 was applied for Fluoro-Blue signals, whereas filter set 15 (BP 546, FT 580, LP 590) was used for Spectrum Orange signals. After changing the format of the images from "TIFF" to "PICT", further processing of the images, including merging and pseudocoloring, was performed with the software package "Gene Join" as described by Ried et al. (1992b).

\section{Results}

Evaluation of G- and R-type patterns of prometaphase spreads obtained after replication banding provided the

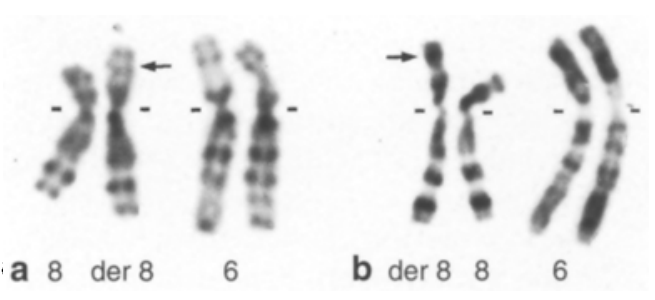

Fig. 1. Partial karyotype (a G-banding, b R-banding; resolution approximately 500 bands per haploid set) of the patient showing a normal and a derived chromosome 8 , together with two normal chromosomes 6 . The derived chromosome 8 shows an unbalanced terminal translocation of two unidentified bands with a breakpoint in band 8 p23 (arrows). On the basis of chromosome banding analyses, the karyotype of the patient was tentatively described as $46, X X,--8,+\mathrm{t}(? ; 8)(?:: 8 \mathrm{p} 23 \rightarrow 8 \mathrm{qter})$ 

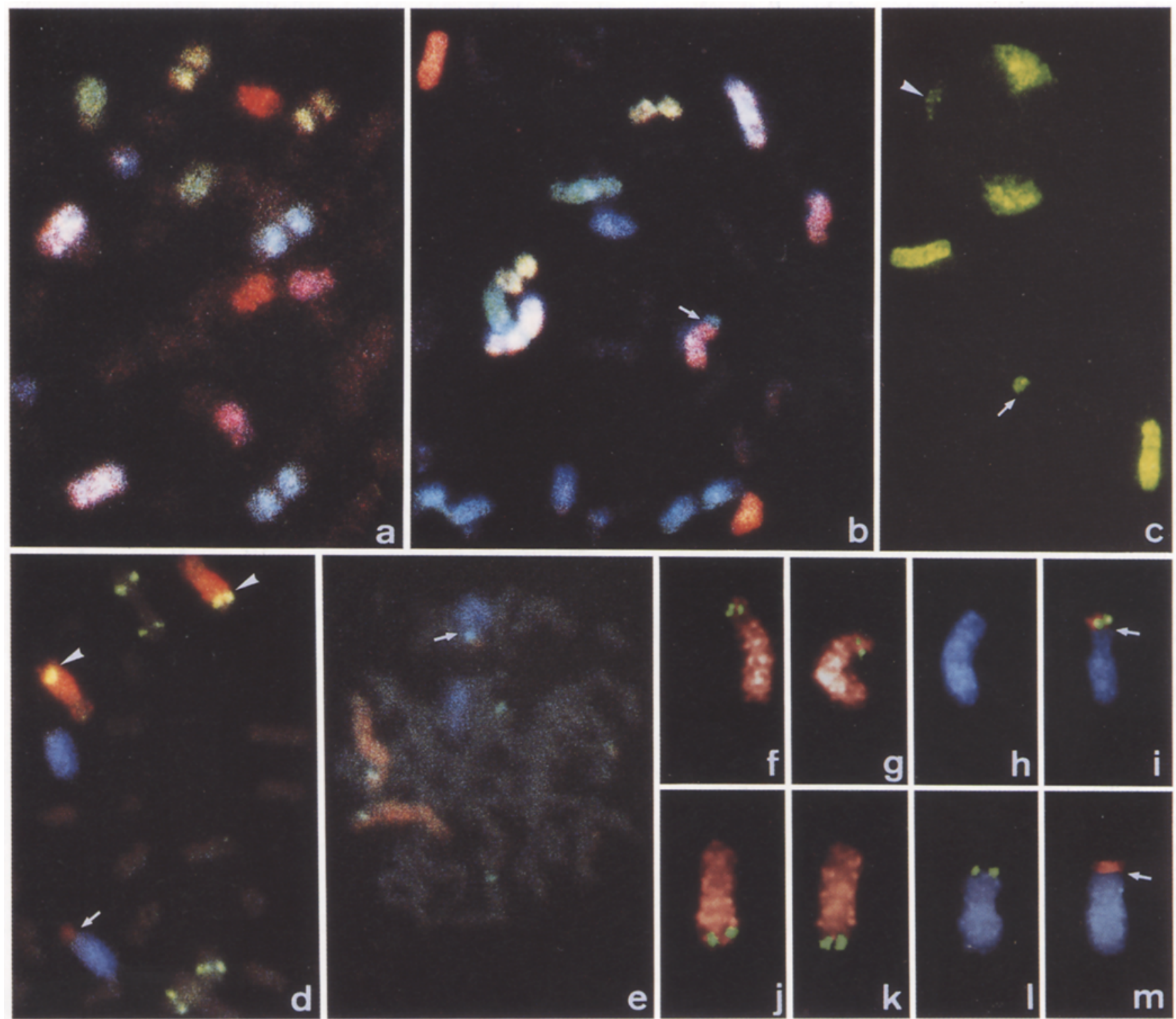

Fig. 2, a Human lymphocyte metaphase spread with a normal karyotype $(46, \mathrm{XY})$ after seven-color painting of chromosomes $1,4,6$, $8,9,12$, and 13 with fluorochrome-labeled plasmid DNA library probes. DNA library probes from flow-sorted human chromosomes were labeled with a single fluorochrome in the case of chromosome 6 (green), chromosome 12 (red) and chromosome 13 (blue). Library probes for chromosomes 1, 8, and 9 were labeled with two fluorochromes (chromosome 1: blue plus green = turquoise; chromosome 8: blue plus red = pink; chromosome 9: green plus red $=$ yellow). Chromosome 4 was painted using a chromosome-4-specific library probe labeled with three fluorochromes (blue plus green plus red $=$ white). $\quad$ b Lymphocyte metaphase spread from the patient after seven-color painting of the chromosome set described in a. The pink of chromosome 8 material and the green of the translocated fragment (arrow) suggest that this fragment originated from chromosome 6. c Partial metaphase spread from the patient after painting of chromosome 6 with a PCR-generated biotinylated chromosome-6-specific library probe. Two normal chromosomes 6 and the translocated fragment in the der(8) (arrow) are delineated (lower). An adjacent interphase nucleus' (upper) shows two normal-sized chromosome domains and a small domain indicating the interphase domain of the translocation fragment (arrowhead). d Partial metaphase spread from the patient after three-color FISH, including painting of chromosome 6 (red) and chromosome 8 (blue). Chromosome painting was combined with FISH of the YAC clone EG8 (green), which maps to 6q25 (arrowheads). Additional YAC signals are visible on both chromosomes 1 at $1 \mathrm{p} 32$ and $1 \mathrm{q} 32$. The arrow points to the trans-

located segment in the der(8). Note that this segment is not delineated by YAC-specific sequences. e Metaphase spread from the patient after painting of chromosomes 6 (red) and 8 (blue) in combination with FISH of YAC clone HTY3103 (green), which maps to $6 \mathrm{p} 22 \rightarrow \mathrm{p} 23$ and $18 \mathrm{q} 23$. The arrow points to a HTY3103-specific signal (green) on the red-painted translocation fragment in the der(8). f-i Red-painted chromosomes 6 (f, g), blue-painted chromosome 8 (h) and the der(8) (i) from another metaphase spread, as described in e, at higher magnification. HTY 3103 signals (green) are seen on the two normal chromosomes 6 (red) and the translocated fragment (red) in the der(8) (i arrow). The results shown in e-i demonstrate that this fragment was derived from $6 \mathrm{p} 23 \rightarrow 6 \mathrm{pter}$. j-m Red-painted chromosomes $6(\mathbf{j}, \mathbf{k})$, blue-painted chromosome 8 (I) and the $\operatorname{der}(8)(\mathbf{m})$ from a metaphase spread of the patient with additional FISH of YAC clone HTY3188 (green signals). This clone maps to $6 \mathrm{q} 27(\mathbf{j}, \mathbf{k})$ and $8 \mathrm{p} 23$ (l). Note: the band localization of the three YACs was confirmed by FISH to G-banded and/or 4,6-diamidino-2-phenyl-indole (DAPI)-banded metaphase spreads (not shown). Hybridization signals from this clone are not seen on the $\operatorname{der}(8)$ chromosome $(\mathbf{m})$ in this and other metaphase spreads. This result independently confirms (1) that the translocation fragment (arrow) does not include material from 6qter, and (2) that a region $8 \mathrm{p} 23 \rightarrow 8$ pter is lacking from the $\operatorname{der}(8)$. Microphotographs on color-slide films shown in a-e were obtained with the Carl Zeiss Axiophot microscope. Single (c) or triple exposures $(\mathbf{a}, \mathbf{b}, \mathbf{d}, \mathbf{e})$ were performed using appropriate filter sets. Images in $\mathbf{f}-\mathbf{m}$ show digitized pseudo-colored chromosome images recorded with a CCD camera and photographed from the screen 
tentative karyotype $46, \mathrm{XX},-8,+\mathrm{t}(? ; 8)(?:: 8 \mathrm{p} 23 \rightarrow 8 \mathrm{qter})$ for the proband, whereas both parents showed normal karyotypes. The origin of the two extra bands on the derivative chromosome 8 could not be identified (Fig. 1). CISS hybridization revealed that chromosome material distal to band 8 p23 in the derivative chromosome 8 was not painted with the chromosome 8 library probe (data not shown). In an attempt to identify the origin of the translocation fragment, combinatorial FISH was applied to paint subsets of up to seven chromosome types in different colors, simultaneously. For multiple color painting of chromosome spreads from the affected girl, two combinations of chromosome-specific library DNA probes covering a total of 11 chromosomes had to be tested until the desired information was obtained. One combination painted chromosomes 2, 3, 7, 8, and 16, but not the fragment (data not shown). The other combination (for chromosomes $1,4,6,8,9,12$, and 13) yielded green painting of the translocated chromosome fragment (Fig.2b). For comparison, Fig. 2a shows a normal lymphocyte metaphase spread $(46, X Y)$ with differently painted chromosomes $1,4,6,8,9,12$, and 13 . Based on the labeling scheme given in Fig. $2 \mathrm{a}$, the green color of the fragment suggested that the material was derived from chromosome 6, which was painted by Spectrum Green only. Accordingly, intense painting of the fragment was recognized using an appropriate FITC filter set. Chromosome 8 material of the marker chromosome was painted by a combination of Spectrum Orange and the coumarin derivative, and it was difficult to decide unequivocally by direct microscopic observation whether the fragment showed additional coloring with either Spectrum Orange (indicating chromosome 9 material) or Fluoro-Blue (indicating chromosome 1 material). For an unequivocal decision between these possibilities, CISS hybridization was separately performed with library probes specific for chromosomes 1, 6, and 9. These experiments demonstrated that the fragment was derived from chromosome 6 (Fig. 2c). With respect to the possibility of a terminal translocation event, YAC clones providing signals on $6 \mathrm{q} 25$ and $6 \mathrm{p} 22 \rightarrow \mathrm{p} 23$, respectively, were chosen in an attempt to narrow down the origin of the fragment. Threecolor FISH was performed with Alu-PCR products from each clone combined with library probes specific for chromosomes 6 and 8 (see legend to Fig. 2 for details of the labeling scheme). YAC clone EG8 localized to $6 q 25$ (and to 1p32 and 1q32) showed the expected signals on chromosomes 1 and 6 of the patient, but did not hybridize to the $8 \mathrm{p}+$ chromosome. In contrast, YAC clone HTY3103, which maps to $6 \mathrm{p} 22 \rightarrow \mathrm{p} 23$ (and 18q23) as confirmed in lymphocyte metaphase spreads of both parents, yielded a strong signal on the $8 \mathrm{p}+$ chromosome, in addition to the expected signals on the normal chromosomes 6 and 18 of the patient (Fig. 2e, i). YAC clone HTY3188, which maps to 6q27, 8p23 (and several other chromosomes, see Materials), hybridized to the expected sites in the normal chromosomes 6 and 8 of the patient (Fig. $2 \mathrm{j}-1$ ), but not to the marker chromosome (Fig. $2 \mathrm{~m}$ ). This finding independently ruled out a terminal $6 \mathrm{q}$ translocation (see above) and at the same time indicated the loss of terminal $8 \mathrm{p}$ material in the marker chromo- some. Based on this analysis the karyotype was refined as: $46, \mathrm{XX},-8,+\mathrm{t}(6 ; 8)(6 \mathrm{pter} \rightarrow 6 \mathrm{p} 22:: 8 \mathrm{p} 23 \rightarrow 8 \mathrm{qter})$.

\section{Discussion}

This study demonstrates the feasibility of using multiple color FISH (Nederlof et al. 1990, 1992; Wiegant et al. 1991; Ried et al. 1992a, b, c) with WCP probes and AluPCR generated probes from various YAC clones to identify rapidly the origin of a small unbalanced chromosome translocation. Using this approach, a trisomy $6 \mathrm{pter} \rightarrow 6 \mathrm{p} 22$ combined with a small terminal deletion $8 \mathrm{p} 23 \rightarrow 8$ pter has been unequivocally demonstrated in a girl with a $8 \mathrm{p}+$ marker chromosome showing severe mental retardation and multiple anomalies. The origin of two additional chromosome bands in the marker chromosome had remained elusive after conventional chromosome banding analysis. In retrospect, several symptoms of the patient, especially blepharoptosis, are consistent with the clinical features described for other patients with a $6 \mathrm{p} 2$ trisomy (for a review, see de Grouchy and Turleau 1984). Additional anomalies, such as a small skull, have been described as a prominent feature of $8 \mathrm{p} 2$ monosomy (de Grouchy and Turleau 1984). It can be expected that the characterization of small unbalanced chromosome segments by multicolor FISH with WCP and region-specific probes will become indispensable in the future for improving the diagnosis of chromosomal syndromes. A detailed discussion of clinical genetic aspects, including the differential diagnosis of other chromosomal syndromes with a similar set of anomalies and malformations will be presented elsewhere (D. Schindler et al., in preparation).

Because of the frequency of terminal translocations or deletions, a series of YAC clones or cosmid clones defining specific chromosome ends will become of particular importance in the development of this technique (Ledbetter 1992a). Telomeric YACs contain subtelomeric repetitive sequences that may be present in variable copy numbers on different chromosomes (IJdo et al. 1992). Such polymorphisms may obscure the interpretation of FISH experiments. To avoid any danger of misinterpretation, it is best to hybridize telomeric YAC clones to chromosome preparations of the patient and both parents.

For broad applications in clinical and tumor molecular cytogenetics, a large set of YAC clones is required covering the whole chromosome complement. Each clone should yield a unique signal on a given chromosome band with high reproducibility both on metaphase chromosomes and in interphase nuclei. The realization of such a goal should allow us to construct multiplex probes optimally tailored for specific diagnostic problems and can be envisaged as an international effort within the ongoing Human Genome Initiative. The number of commercially available probes conjugated with appropriate haptens or even directly with fluorochromes is steadily increasing, and will greatly facilitate the introduction of multiple color FISH in cytogenetic laboratories lacking the equipment or the experience required for probe preparation and complex detection schemes (Ledbetter 1992b). By applying FISH with multiplex probes prepared from 
sets of YAC clones, we have started to construct new types of colored chromosome staining patterns, termed chromosomal bar codes (Lengauer et al. 1993).

In addition to improved studies of chromosomal syndromes, the strategy presented in this paper should become particularly useful for the rapid identification of multiple rearrangements in tumor cells. When the number of metaphase spreads available for analysis is severely limited, which is often the case in tumor material, two possibilities may be considered in order to perform a comprehensive analysis. (1) Digital fluorescence microscopy based on ratio imaging provides the possibility of increasing further the number of targets that can be simultaneously painted in different colors (Nederlof et al. 1992; Dauwerse et al. 1992; du Manoir et al. 1993). Ratio imaging with four spectrally separable fluorochromes should, in near future, allow the discrimination of all 24 human chromosome types in different colors in a single FISH experiment. (2) Several FISH experiments may be carried out with the same few metaphase spreads, if different probe sets are applied sequentially (Heslop-Harrison et al. 1992).

A new approach to studying genetic imbalances, termed comparative genomic hybridization (CGH), has recently been described (Kallioniemi et al. 1992; du Manoir et al. 1993). For CGH, genomic DNA prepared from a clinical or tumor specimen (test genome) is modified with a hapten (e.g. with biotin), whereas genomic DNA prepared from cells with normal chromosome complements $(46, \mathrm{XY}$ or $46, \mathrm{XX})$ is labeled with a different hapten (e.g. digoxigenin). Labeled test and control genomic DNA are mixed $(1: 1)$, and used as a probe to paint metaphase spreads with normal chromosome complements $(46, X X$ or $46, X Y)$. Following detection of the two haptens with two fluorochromes, such as FITC and tetraethylrhodamine isothiocyanate (TRITC), the ratios of FITC/TRITC fluorescence intensities can be measured along each normal chromosome. These ratios reflect the copy numbers of chromosomes or chromosomal segments present in the test genome (Kallioniemi et al. 1992; du Manoir et al. 1993; our own unpublished data). Whereas CGH provides a powerful approach for the detection of genetic imbalances, the sensitivity of $\mathrm{CGH}$ in detecting partial chromosome gains and losses is not yet clear (Kallioniemi et al. 1992; du Manoir et al. 1993).

Multiple color FISH with WCP probes and subregional DNA probes ideally complements chromosome banding and CGH. It can be used for the independent confirmation of findings obtained by the two other approaches and to study the breakpoints involved in chromosomal rearrangements at much higher resolution. Finally, it should be emphasized that both chromosome banding and multiple color FISH with multiplex probes can be used to identify marker chromosomes at the single cell level, and thus can be applied to detect clonal aberrations present in only a few cells. In contrast, CGH can only detect genetic imbalances consistently occurring in the majority of cells from which test genomic DNA is prepared. It provides neither information on the presence of balanced chromosome rearrangements nor details of the way in which chromosome segments involved in gains and losses are arranged in marker chromosomes.
Acknowledgements. We gratefully acknowledge the expert assistance of Klaus Steinlein in chromosome replication banding analyses of the patient and her parents, Brigitte Schoell for help in the preparation of probe DNAs, and Verona Weickardt for photographic work. This work was supported by grants from the European Community (GENO-CT91-0029) and the "Forschungsschwerpunktprogramm des Landes Baden-Württemberg an den Universitäten".

\section{References}

Collins CC, Kuo WL, Segraves R, Fuscoe JC, Pinkel D, Gray JW (1991) Construction and characterization of plasmid libraries enriched in sequences from single human chromosomes. Genomics 11:997-1006

Dauwerse JG, Wiegant J, Raap AK, Breuning MH, Ommen GJB van (1992) Multiple colors by fluorescence in situ hybridization using ratio-labelled DNA probes create a molecular karyotype. Hum Mol Genet 1:593-598

Dutrillaux B, Couturier J, Richer CL, Viegas-Pequinot E (1976) Sequence of DNA replication in $277 \mathrm{R}$ - and Q-bands of human chromosomes using a BrdU treatment. Chromosoma 58:5161

Grouchy J de, Turleau C (1984) Clinical atlas of human chromosomes, 2nd edn. Wiley, New York

Heslop-Harrison JS, Harrison GE, Leitch IJ (1992) Reprobing of DNA:DNA in situ hybridization preparations. Trends Genet 8 : 372-373

IJdo JW, Lindsay EA, Wells RA, Baldini A (1992) Multiple variants in subtelomeric regions of normal karyotypes. Genomics $14: 1019-1025$

Johnson GD, Nogueira Araujo GM de C (1981) A simple method of reducing the fading of immunofluorescence during microscopy. J Immunol Methods 43:349-350

Kallioniemi A, Kallioniemi OP, Sudar D, Rutovitz D, Gray JW, Waldman F, Pinkel D (1992) Comparative genomic in situ hybridization for molecular cytogenetic analysis of solid tumors. Science 258:818-821

Ledbetter DH (1992a) Minireview: cryptic translocations and telomere integrity. Am J Hum Genet 51:451-456

Ledbetter DH (1992b) The "colorizing" of cytogenetics: is it ready for prime time? Hum Mol Genet 1:297-299

Lengauer C, Green ED, Cremer T (1992) Fluorescence in situ hybridization of YAC clones after Alu-PCR amplification. Genomics $13: 826-828$

Lengauer C, Speicher MR, Popp S, Jauch A, Taniwaki M, Nagaraja $\mathrm{R}$, Riethman HC, Donis-Keller H, d'Urso M, Schlessinger D, Cremer T (1993) Chromosomal bar codes produced by multicolor fluorescence in situ hybridization with multiple YAC clones and whole chromosome painting probes. Hum Mol Genet 2:505-512

Lichter P, Cremer T (1992) Chromosome analysis by non-isotopic in situ hybridization. In: Rooney DE, Czepulkowski BH (eds) Human cytogenetics - a practical approach, vol F, 2nd edn. IRL Press, Oxford, pp 157-192

Lichter P, Boyle AL, Cremer T, Ward DC (1991) Analysis of genes and chromosomes by nonisotopic in situ hybridization. Genet Anal Techn Appl 8:24-35

Manoir S du, Speicher MR, Joos S, Schröck E, Popp S, Döhner H, Kovacs G, Robert-Nicoud M, Lichter P, Cremer T (1993) Detection of complete and partial chromosome gains and losses by comparative genomic in situ hybridization. Hum Genet 90: 590-610

Nederlof PM, Flier S van der, Wiegant J, Raap AK, Tanke HJ, Ploem JS, Ploeg M van der (1990) Multiple fluorescence in situ hybridization. Cytometry $11: 126-131$

Nederlof PM, Flier S van der, Vrolijk J, Tanke HJ, Raap AK (1992) Fluorescence ratio measurements of double-labeled probes for multiple in situ hybridization by digital imaging microscopy. Cytometry 13:839-845 
Pinkel D, Gray JW, Trask B, Engh G van den, Fuscoe J, Dekken H van (1986) Cytogenetic analysis by in situ hybridization with fluorescently labeled nucleic acid probes. Cold Spring Harb Symp Quant Biol 51:151-157

Ried T, Baldini A, Rand TC, Ward DC (1992a) Simultaneous visualization of seven different DNA probes by in situ hybridization using combinatorial fluorescence and digital imaging microscopy. Proc Natl Acad Sci USA 89: 1388-1392

Ried T, Landes G, Dackowski W, Klinger K, Ward DC (1992b) Multicolor fluorescence in situ hybridization for the simultaneous detection of probe sets for chromosomes 13,18,21, $\mathrm{X}$ and $\mathrm{Y}$ in uncultured amniotic fluid cells. Hum Mol Genet 1:307313

Ried T, Lengauer C, Cremer T, Wiegant J, Raap AK, Ploeg M van der, Groitl P, Lipp M (1992c) Specific metaphase and inter- phase detection of the breakpoint region in 8q24 of Burkitt lymphoma cells by triple-color fluorescence in situ hybridization. Genes Chrom Cancer 4:1-6

Sambrook J, Fritsch EF, Maniatis T (1989) Molecular cloning: a laboratory manual, 2nd edn. Cold Spring Harbor Laboratory, Cold Spring Harbor, NY

Telenius H, Pelmear AH, Tunnacliffe A, Carter NP, Behmel A, Ferguson-Smith MA, Nordenskjöld M, Pfragner R, Ponder BAJ (1992) Cytogenetic analysis by chromosome painting using DOP-PCR amplified flow-sorted chromosomes. Genes Chrom Cancer 4:257-263

Wiegant J, Ried T, Nederlof PM, Ploeg $M$ van der, Tanke HJ, Raap AK (1991) In situ hybridization with fluoresceinated DNA. Nucleic Acids Res 19:3237-3241 